\title{
Radical approach for centrally located echinococcal cysts of the liver
}

\begin{abstract}
Introduction: Liver echinococcosis is still an endemic disease in Balkans. PAIR is actually considered the safest therapeutically approach for uncomplicated type I and II cysts, while surgery is indicated for type III or complicated cysts. It's commonly believed that radical surgery could be considered the most appropriate treatment for hydrated disease, but with higher risk of morbidity, much more for centrally located cysts, especially in low-resources hospitals.
\end{abstract}

Aim of the study: According with the tendency in western countries and within a training project leaded by Veneto Region - Italy, we started in cooperation with the Division of Surgery of Peja Hospital Kosovo a prospective study to assess the safety and effectiveness of radical surgery also for centrally located cysts, when PAIR was contraindicated.

Materials and Methods: From Feb 2006 to Apr 2010, 10 consecutive patients (6 Female, 4 males, mean age $31,3 \pm 14$ yrs, range 8-54) with 16 cysts were treated for echinococcal liver disease in Surgical Division of Peja Hospital, Kosovo. All patients received Albendazole before operation and in the perioperative period. Medical therapy was discontinued the day after operation. One patient had recurrent echinococcal cysts after previous operation of partial cystectomy and T-tube placement in the biliary duct. Patients were classified to have peripherical $(n=1)$ or centrally $(n=9)$ located cysts when at least one of them was in close relationship with the main vascular and biliary structures. Other than laboratory tests, preoperative evaluation included in all patients abdominal ultrasound and CT scan.

Results: Nine pts with 15 cysts underwent radical surgery and 1 patient underwent non radical operation ( 1 subtotal pericystectomy). Four of them had multiple cysts. Mean operative time was $236 \mathrm{~min}$. Mean postoperative stay was 5, 2 $\pm 2,52$ days (range 3-10). No intra or postoperative complications were observed after radical surgery. A bile leak that healed spontaneously after 9days occurred in patient with subtotal pericystectomy. Mean follow-up is longer than 3years.

Discussion: Therapeutic choices for echinococcal liver disease depend from factors related to patient, cyst, availability of resources and physician. Radical surgery is considered the treatment of choice because of complete elimination of the parasite and the residual cavity, minimizing the risk of recurrence. This approach, especially for central cysts, must be balanced with the risk of complications, but it's the preferred one in Centers with expertise in liver surgery and it was possible in $93 \%$ of cases in this series. In our experience mean operative time was prolonged because of meticulous dissections of cysts from surrounding tissue and vessels. It was always possible to dissect the main veins from pericystium, while portal triad structures were more often eroded by the cysts and needed to be sutured. Mean follow-up is more than 3years and no recurrences were observed until now.

Conclusion: Open radical surgery can be considered a reliable therapeutic option for liver hydatidosis for centrally located cysts also in poor resources hospital. Patients with echinococcal cysts need to be referred to Centers in which therapy is evaluated and treated in a multidisciplinary team with specific expertise in liver surgery.

Keywords: pericystectomy, liver hydatidosis, radical, echinococcal cysts, pericyst
Volume 3 Issue I - 2017

Recordare A,' Shala F, Dreshaj R, ${ }^{2}$ Vrella B, ${ }^{2}$ Berisha $\mathrm{S},{ }^{2}$ Loxha $\mathrm{N},{ }^{2}$ Muhaxheri $\mathrm{S},{ }^{3}$ Kastrati $\mathrm{B},{ }^{4}$ Kingji $\mathrm{H}^{4}$

IIV Division of Surgery, Treviso Regional Hospital, Italys

${ }^{2}$ Department of Surgery, Peja Regional Hospital, Italy ${ }^{3}$ Department of Radiology, Peja Regional Hospital, Italy ${ }^{4}$ Department of Anesthesia and Intensive Care, Peja Regional Hospital, Italy Correspondence: Recordare A, Department of Surgery,
Treviso Regional Hospital, Kosovo, Italy,

Email alf.recordare@gmail.com

Received: September 18, 2016 | Published: January 23, 2017
Abbreviations: CT, computerized tomography; US, ultrasound

\section{Introduction}

Hydatidosis is still a major health problem in developing countries and endemic disease in Balkans. Infection with the larval stage of the dog tapeworm Echinococcosis granulosus is the most commonly occurring type of echinococcosis. ${ }^{1,2}$ The liver is affected in about $80 \%$ of cases, although hydatid cysts may develop in almost any organ of the body. ${ }^{3}$ Cyst characteristics differ according to the evolutionary phase of the parasite and can be carefully described by the ultrasound (US) images. US classification for the hydatid cysts of the liver was standardized by the WHO-IWGE, ${ }^{4}$ with the aim to be helpful to select the proper therapeutic approach. Medical treatment has been proven to be effective against larval E. granulosus, if used in combination with PAIR or surgery. PAIR is a minimally invasive technique that consists 
of percutaneous puncture of the cyst, aspiration of the contents for injection of scolicidal agent and finally, re-aspiration of the full contents. ${ }^{5}$ This approach is becoming more widely used and actually PAIR plus drug therapy represents the first recommended approach, mainly for uncomplicated cysts with a limited number of daughter cysts, with diameter until $7-8 \mathrm{~cm}$, for the reported low complication rate and because it don't hamper surgical treatment of refractory cysts. The role of surgery is progressively narrowing, being confined to the most complicated cases that require more surgical expertise. ${ }^{6-9}$ Surgery needs to be effective to inactivate scolices, preventing recurrence and spillage into the abdomen and to obliterate the residual cavity in order to decrease the rate of post-operative complications. There is a wide range of procedures and all of them are studied to obtain these purposes. They are grossly divided between radical and not radical procedures.

It's very well known that radical procedures give the best results, but they are more complex and require specific expertise. In fact, it is generally believed that more radical is the intervention, higher the operative risk, but lower the recurrence rate, whereas to a more conservative approach corresponds a lower operative risk of mortality but an higher recurrence and complication rate. Moreover, more the cyst is in close relationship with the main vascular and biliary structures, it means centrally located, more the radical excision is technically demanding and the expected rate of intra-operative complications higher. On the other hand, morbidity and mortality for liver surgery is going to decrease ${ }^{10}$ and limited liver resections for benign disease is associated with very low morbidity. ${ }^{11}$ This is why high volume Centres for hepato-biliary surgery in western countries shifted their approach to the radical one from many years ${ }^{6-9,12-14}$ and this trend is slowly spreading in the other countries. In spite of this trend, looking at the surgical series, especially in endemic countries, the most commonly worldwide performed operation is still the non radical partial cystectomy. ${ }^{6-9}$ Within a training project in surgery leaded by Veneto Region- Italy, we started a prospective study to assess the safety and effectiveness of a surgical radical approach for echinococcal cysts, when PAIR was contraindicated, also for centrally located cysts, in a poor resources Hospital.

\section{Materials and methods}

Ten consecutive patients with liver echinococcal cysts that didn't meet criteria for PAIR treatment (M:4, F:6), with a mean age of $31,3 \pm 14$ years (range $8-54$ ) were prospectively enrolled and operated in the Department of Surgery of Peja Hospital (Kosovo) from Feb 2006 until Apr 2010. Preoperative evaluation of the patients included blood tests (complete blood count, liver function tests and antiechinococcus antibody testing) abdominal US and computerized tomography (CT). Five patients had single and 5 patients multiple cysts for a total number of 16 cysts. Three patients had previous surgery for hydatidosis: 1 patient had partial cystectomy 3 years before and come for recurrence, another patient with cysto biliary fistula underwent an unclear attempt to remove the cyst and cholecystectomy 3 months before (Figure 1) and another patient was previously operated for lung echinococcosis. One patient underwent PAIR 1 year before operation. According to the relationship with the main vascular and biliary structures, cysts were classified as peripheral $(n=4,25 \%)$ or central $(n=12,75 \%)$, when they had very close relationship with the main vessels. CT scan was considered mandatory for an adequate operative plan. RMN and ERCP were not available in the country during the study period. An intraoperative cholangiogram was obtained only in case of clinical history or laboratory tests alteration suggestive for bile duct obstruction. All patients were treated with Albendazole before surgery and in the perioperative period. Treatment was stopped immediately after surgery if during the operation the cysts were not opened. To prevent accidental spillage into the abdomen, the operative field was protected with gauzes soaked by hypertonic saline solution. Parenchymal transection was performed by clamp-crush technique. During the operation, afferent blood vessels and biliary ducts were ligated between the pericyst and the normal liver. Vessels less than $2 \mathrm{~mm}$ in diameter were sealed using humid bipolar coagulation (Figure 2). No other devices were available to transect the liver, or glues to treat the cut surface.

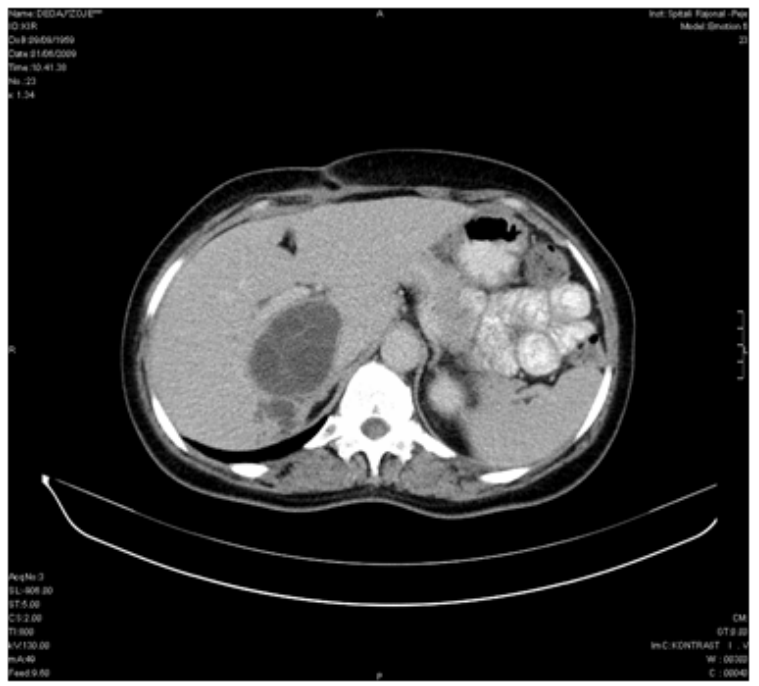

Figure I CT scan show a cyst situated between the portal confluence and the caval vein. Patient suffered jaundice and pain from cysto-biliary fistula.

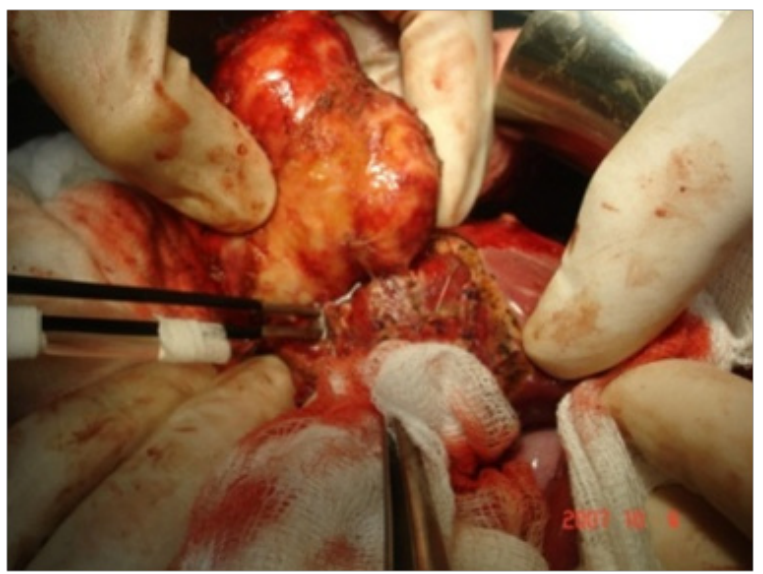

Figure 2 Vessels with diameter less than $2 \mathrm{~mm}$ were sealed using humid bipolar coagulation. The other vessels were traditionally dissected and ligated.

\section{Results}

Sixteen cysts were excised in 10 patients. Nine patients underwent radical surgery; 5 had multiple cysts and were submitted to hepatic resection (1 right and 2 left hepatectomy) or multiple total pericystectomy with closed cyst. Four patients with single cyst underwent total pericystectomy with closed cyst. Only 1 patient underwent a non-radical operation and a subtotal pericystectomy was performed. In this case the cyst was opened and a small piece of the pericystium was left in place close to the right branch of the portal vein and the bile duct. A cysto-biliary fistula with the right posterior 
bile duct was sutured after washing of the biliary tree. Only in one case intermittent Pringle maneuver was used to control bleeding. In the other cases the inflow was controlled by selective clamping, if necessary. In 2cases previous clamping at the origin of the right hepatic vein was performed before dissection of the vein from the pericystium. Intraoperative ultrasound was necessary only in one case of subtotal pericystectomy. It was always possible to dissect the main veins from the pericystic wall (Figure 2-4), while it was necessary

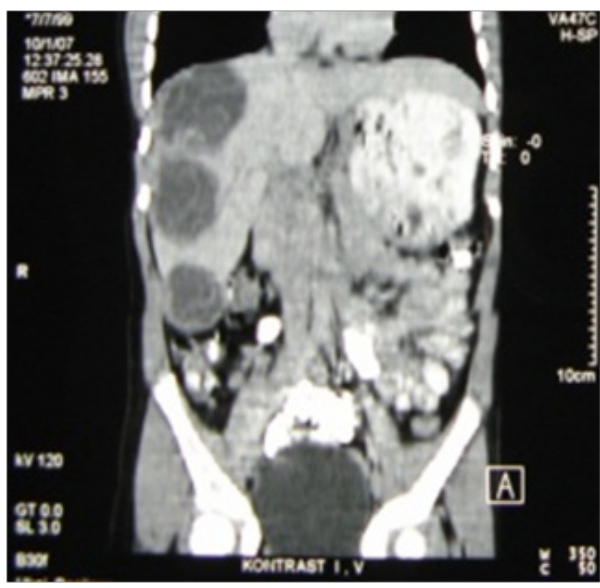

to sacrifice the structures of the portal triad when they were fused in the pericyst. Mean operative time was $236 \mathrm{~min}$. All patients were extubated at the end of the procedure. Mean postoperative stay was $5.2 \pm 2,52$ days (range 3-10), with no mortality and only 1 low output biliary fistula in the case of subtotal pericystectomy that healed after 9days. Postoperative stay was longer for children because they lived far from the hospital. Mean follow up is higher than 3years and until now no recurrence were observed (Figure 5).

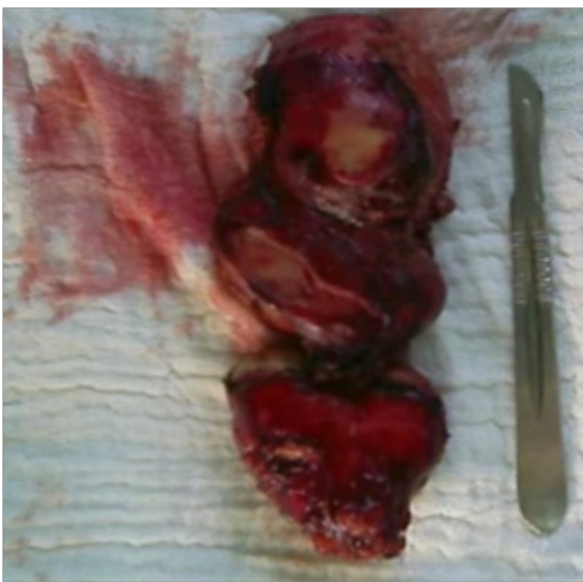

Figure 3 Multiple hydatid cysts in the right lobe of the liver in 9 years old girl. Multiple total pericystectomy with closed cysts was performed.
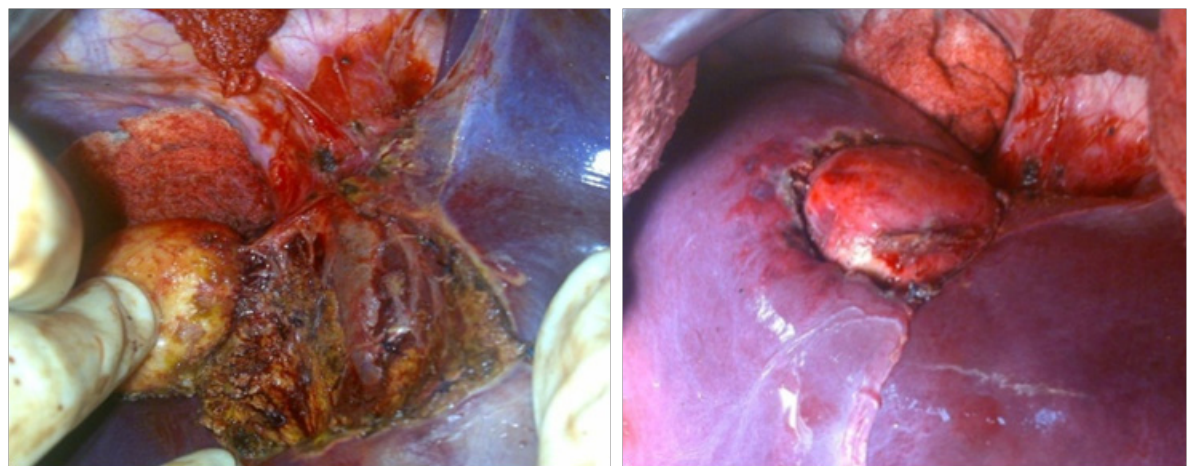

Figure 4 After adequate exposure, it's always possible to dissect the cyst from main hepatic veins. In this case a cyst of segment IV-VIII is dissected from middle hepatic vein
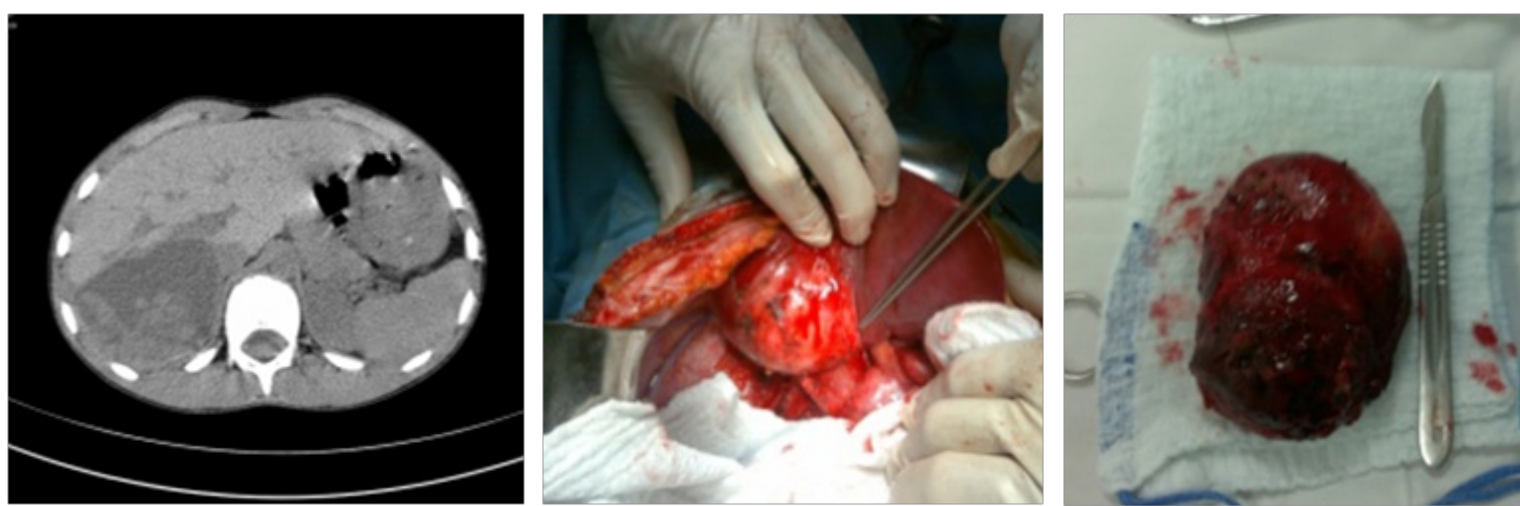

Figure 5 Total pericystectomy for gyant cyst of the right liver lobe in close relationship with the caval vein and the right portal branch. This patient underwent PAIR I year before surgery.

\section{Discussion}

Treatment of liver echinococcosis depends not only from the characteristics and location of the cysts, but also from the available resources and the physician. In the PAIR era, surgical indications are becoming more restricted (Table 1). Liver echinococcosis is a benign disease but, from a surgical point of view, it can show a wide range of difficulties depending on the location of the cysts, their relationship with the main vascular and biliary structures, previous surgery and concomitant diseases. ${ }^{6-12,15}$ Non radical procedures 
performed for hydatid cysts vary from simple puncture and aspiration of the cyst to unroofing associated with various procedures for the management of residual cavity. These are technically easier and safer but are associated with high incidence of local recurrence and cavityrelated complications at rates higher than $10 \% .{ }^{7,13}$ Radical procedures include pericystectomy and hepatic resection. Total pericystectomy can be performed either with the "open" or the "closed-cyst" method. In the open technique, the cyst is opened, the contained material is removed, anti-scolecidal substances are very often infused and then the pericyst is removed. In the closed-cyst method, en block pericystectomy is performed with no other manipulations. During the pericyst's removal, afferent blood and biliary vessels are ligated in order to prevent haemorrhage or postoperative bile leakage. These procedures are more technically difficult and time spending but allow the complete removal of the parasite, avoid the formation of a residual cavity and allow identifying daughter cysts adjacent to the main wall. There are many experiences that clearly show that radical surgery gives superior results and must be considered the ideal approach in terms of recurrence and post-operative course, when feasible. ${ }^{6-9}$ What means feasible is debatable. It's suggested that the operation is to be avoided for central cysts, impinging on the major hepatic veins, the inferior vena cava and close to the liver hilum, because the risk of profuse bleeding, so that the percentage of radical operations vary in the different series but rarely reaches $50 \%,{ }^{6-12,16}$ with only one exception ${ }^{14}$ and this percentage is closely related to the expertise in liver surgery of the surgical team. In recent years an increased number of cases were treated by celioscopy, but, as in open surgery, results are mainly related to the type of operation and the characteristic of the cysts, more than the surgical approach (Figure 6). ${ }^{17,18}$

Table I Actual Indications to surgery for liver hydatidosis

$$
\begin{aligned}
& \text { Large Cysts or with Multiple Daughter Cysts } \\
& \text { Superficial Cysts With Risk of Rupture } \\
& \text { Infected Cysts } \\
& \text { Cysts Communicating with the Biliary Tree } \\
& \text { Cysts Exerting Pressure on other Vital Organs }
\end{aligned}
$$

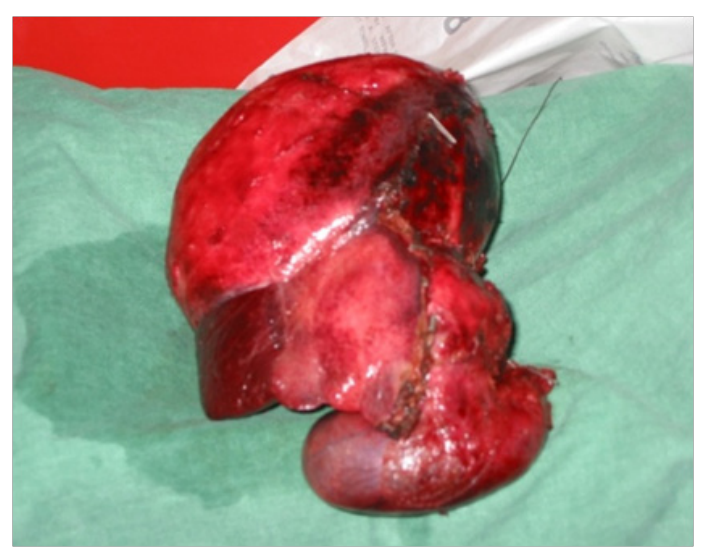

Figure 6 Right Hepatectomy for multiple echinococcal cysts in the right lobe. Also the wall of the gallbladder was involved by the parasitic cyst.

In order to evaluate which cyst can be candidate to radical surgery and the technical challenges of a radical removal, we think that there is the need to take into account the different biological behavior of the cysts in front of the main hepatic veins or the portal triad structures, especially the arterial and biliary branches. It has a very big impact on the possibility to perform a radical operation. In our experience it was always possible to detach the pericyst from the main hepatic veins, especially if their lumen is detectable in CT scan, while it was much more difficult to spare the structures of the portal triad when they are fused in the pericyst. It's easily understandable considering the biology of the cyst. The cyst is nourished from the arterial and maybe portal vessels and we found that these vessels are more easily incorporated in the pericyst, until they disappear in the connective tissue, while the biliary ducts erode the pericyst. It's very well known the tendency of fistulisation of the biliary ducts within the cyst. It is demonstrated that the presence of a significant biliary fistula is related to the diameter of the cyst ${ }^{23}$ and to their position close to the hepatic hylum. ${ }^{19}$ It means that the presence of a fistula is linked with the probability that the cyst find, in the growth process, a large bile duct. A very interesting study on the histopathology of the pericyst ${ }^{20}$ showed the surprisingly constant finding of small areas of fibrinoid necrosis where the fistula is going to develop. Furthermore, the appearance of bile from the drain is very common after 24-48hours after percutaneous puncture of large diameter hydatid cyst, as the tension inside the cyst diminish as it is quite frequent the development of "late" biliary fistulas after not radical surgery. ${ }^{21}$

The behaviour of major veins is different. They are more often dislodged than infiltrated and maintain their identity in the pericystium. This circumstance is also consistent with the observation that rupture of the cyst in the main hepatic veins is an extremely rare event ${ }^{16}$ while, more often, the veins are simply compressed, until the development of a Budd-Chiari syndrome that reverse after operation..$^{22}$ These observations indirectly demonstrate the difficulties to isolate and preserve, during the surgical procedure of pericystectomy, the arterial and biliary branches more than the hepatic veins. With the actually available preoperative imaging, careful evaluation of CT and/or NMR allows to plan, just before operation, the structures that can be preserved or would eventually been sacrificed during surgery. It's a very crucial step in the preoperative planning. In this series the percentage of radically treated cysts exceed $93 \%$, if we like to consider a subtotal pericystectomy a non-radical procedure. When the cyst is fistulised in the main branches of the bile ducts, subtotal pericystectomy is an alternative to partial cystopericystectomy and the closure of the fistula can be secured from the internal part of the pericystic wall. This approach is more reasonable and avoid the possible morbidity linked to the opening of the bile duct Liver resection is rarely required. Although this method seems to be the most complete treatment for hydatid disease, it is indicated when cysts have destroyed an entire lobe or segment, thus compressing the healthy parenchyma interrupting the bile ducts, or there is the need to resect draining zones of fistulised bile ducts. In such cases left or right hepatectomy or segmentectomy can be performed..$^{12,23-26}$

\section{Conclusion}

This experience suggests that radical surgery, also for centrally located echinococcal cysts, is a viable therapeutic option also in hospitals with limited financial resources. Very encouraging results can be obtained also without the availability of the commonly worldwide used intraoperative devices for liver surgery, so the difference in the percentage of radically treated cases needed to be increased also in these countries, but from a trained surgical team. Moreover, the increasing indications to PAIR (that have better results than non radical surgery) will increase the percentage of complicated cases in the surgical series that will require more expertise in liver surgery and we advice the organization of referral Center in endemic 
countries with adequate skills to manage the broad spectrum of the disease. Nowadays it's not anymore the matter to compare surgery to PAIR, because of the widely accepted difference in indications, but we think that it's important to consider that, according to the compliance of the patient and the characteristics of the cyst, the need to continue drug therapy and the need for close follow up are arguments in favor of radical surgery in front of borderline indications to PAIR especially if it requires to leave a drain in place for long time.

\section{Acknowledgements}

None.

\section{Conflict of interest}

Author declares that there is no conflict of interest.

\section{References}

1. Mihmanli M, Idiz UO, Kaya C, et al. Current status of diagnosis and treatment of hepatic echinococcosis. World J Hepatol. 2016;8(28):1169-1181.

2. Rossi P, Tamarozzi F, Galati F, et al. The first meeting of the European Register of Cystic Echinococcosis (ERCE). Parasit Vectors. 2016;9:243.

3. Brunetti E, Praticò L, Neumayr A, et al. Update on Treatment for Cystic Echinococcosis of the Liver. Curr Treat Options Infect Dis. 2016;8(3):153-164.

4. Brunetti E, Kern P, Vuitton DA. Expert consensus for the diagnosis and treatment of cystic and alveolar echinococcosis in humans. Acta Trop. 2010;114(1):1-16.

5. Giorgio A, Di Sarno A, De Stefano G, et al. Percutaneous treatment of Hydatid Liver Cyst. Recent Pat Antiinfect Drug Discov. 2009;4(1):29-36.

6. Secchi MA, Pettinari R, Mercapide C, et al. Surgical management of liver hydatidosis: a multicentre series of 1412 patients. Liver Int. 2010;30(1):85-93.

7. Buttenschoen K, Carli Buttenschoen D. Echinococcus granulosus infection: the challenge of surgical treatment. Langenbecks Arch Surg. 2003;388(4):218-230.

8. Tasev V, Dimitrova V, Draganov K, et al. Hepatic echinococcosis: radical or conservative surgical treatment. Khirurgiia (Sofia). 2002;58(2):10-13.

9. Aydin U, Yazici P, Onen Z, et al. The optimal treatment of hydatid cyst of the liver: radical surgery with a significant reduced risk of recurrence. Turk J Gastroenterol. 2008;19(1):33-39.

10. House MG, Ito H, Gönen M, et al. Survival after hepatic resection for metastatic colorectal cancer: trends in outcomes for 1,600 patients during two decades at a single institution. J Am Coll Surg. 2010;210(5):744-752.

11. Erdogan D, Busch OR, Gouma DJ, et al. Morbidity and mortality after liver resection for benign and malignant hepatobiliary lesions. Liver Int. s2009;29(2):175-180.
12. De Werra C, Condurro S, Tramontano S, et al. Hydatid disease of the liver: thirty years of surgical experience. Chir Ital. 2007;59(5):611-625.

13. Nardo B, Patriti A, Piazzese E, et al. Radical surgical treatment of recurrent hepatic hydatidosis. Hepatogastroenterology. 2003;50(53):1478-1481.

14. Botrugno I, Gruttadauria S, Li Petri S, et al. Complex hydatid cysts of the liver: a single center's evolving approach to surgical treatment. Am Surg. 2010;76(9):1011-1015

15. Prousalidis J, Kosmidis $\mathrm{CH}$, Fahantidis E, et al. Surgical treatment of multiple cystic echinococcosis. HPB (Oxford). 2004;6(2):110-114.

16. Coskun I, Esentürk M, Yörük Y. The rupture of hepatic hydatid disease into the right hepatic vein and bile ducts: a case report. Hepatogastroenterology. 1996;43(10):1006-1008.

17. Busić Z, Lemac D, Stipancić I, et al. Surgical treatment of liver echinococcosis--the role of laparoscopy. Acta Chir Belg. 2006;106(6):688-691.

18. Sharma D, Babu R, Borgharia S, et al. Laparoscopy for liver hydatid disease: where do we stand today? Surg Laparosc Endosc Percutan Tech. 2009;19(6):419-423

19. Kayaalp C, Bostanci B, Yol S, et al. Distribution of hydatid cysts into the liver with reference to cystobiliary communications and cavity-related complications. Am J Surg. 2003;185(2):175-179.

20. Stamm B, Fejgl M, Hueber C. Satellite cysts and biliary fistulas in hydatid liver disease. A retrospective study of 17 liver resections. Hum Pathol. 2008;39(2):231-235.

21. Sönmez K, Karabulut R, Türkyilmaz Z, et al. Clear cystic fluid in hepatic hydatidosis does not rule out communication between cysts and the biliary system. Adv Ther. 2007;24(2):291-295.

22. Bedioui H, Nouira K, Ayadi S, et al. Budd-Chiari syndrome secondary to hepatic echinococcosis. Gastroenterol Clin Biol. 2007;31(8-9 Pt 1):721-724.

23. Yagci G, Ustunsoz B, Kaymakcioglu N, et al. Results of surgical, laparoscopic, and percutaneous treatment for hydatid disease of the liver: 10 years experience with 355 patients. World J Surg. 2005;29(12):1670-1679.

24. El Malki HO, El Mejdoubi Y, Souadka A, et al.Predictive model of biliocystic communication in liver hydatid cysts using classification and regression tree analysis. BMC Surg. 2010;10:16.

25. Priego P, Nuño J, López Hervás $P$, et al. Hepatic hydatidosis. Radical vs. conservative surgery: 22years of experience. Rev Esp Enferm Dig. 2008;100(2):82-85.

26. Unalp HR, Baydar B, Kamer E, et al. Asymptomatic occult cysto-biliary communication without bile into cavity of the liver hydatid cyst: a pitfall in conservative surgery. Int J Surg. 2009;7(4):387-391. 